\title{
AN OPTICAL OFFGAS SENSOR NETWORK INCORPORATING A HG CAVITY RINGDOWN SPECTROMETER AND IR DIODE LASERS
}

\author{
Final Technical Progress Report
}

\author{
Work Performed Under Contract: \\ DE-FG26-06NT42686 \\ Dr. George P. Miller \\ Department of Physics and Engineering Physics \\ The University of Tulsa \\ 600 S College Avenue \\ Tulsa, OK 74104 \\ Tel: (918) 631-3021, FAX: (918) 631-2995 \\ Email: gpmiller@utulsa.edu
}

Submitted To:

U. S. Department of Energy

Morgantown Energy Technology Center

Morgantown, West Virginia

COR: Susan Maley 


\section{Disclaimer:}

This report was prepared as an account of work sponsored by an agency of the United States Government. Neither the United States Government nor any agency thereof, nor any of their employees, makes any warranty, express or implied, or assumes any legal liability or responsibility for the accuracy, completeness, or usefulness of any information, apparatus, product, or process disclosed, or represents that its use would not infringe privately owned rights. Reference herein to any specific commercial product, process, or service by trade name, trademark, manufacturer, or otherwise does not necessarily constitute or imply its endorsement, recommendation, or favoring by the United States Government or any agency thereof. The view and opinions of authors expressed herein do not necessarily state or reflect those of the United States Government or any agency thereof. 


\section{Abstract:}

A multi-element cavity ringdown system was evaluated with the objective of developing an intelligent sensor network to be incorporated into the control systems for advanced coal combustion facilities. Using a combination of a YAG-pumped dye laser and a tunable NIR/IR laser a dual cavity was constructed and a labview program was developed to provide multi-channel, real-time data to permit the real-time monitoring of typical exhaust emission gases, (for example: $\mathrm{CO}_{2}, \mathrm{SO}_{2}$, and mercury) of concern to the next generation of coal-powered facilities.

\section{Table of Contents}

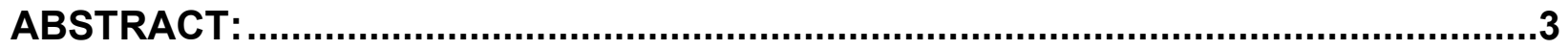

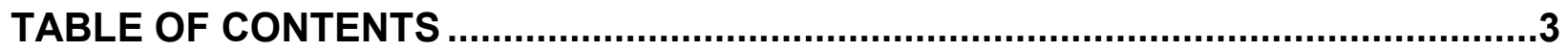

EXECUTIVE SUMMARY ................................................................................4

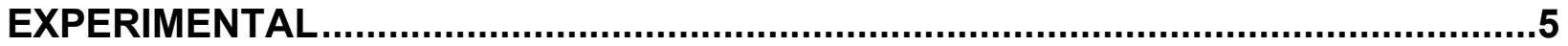

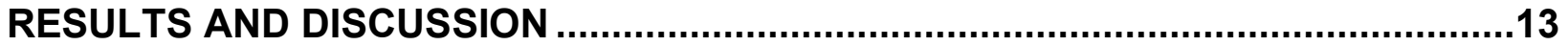

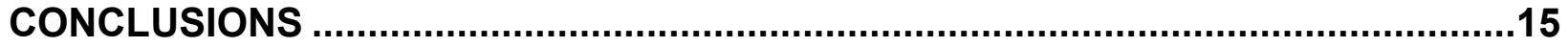

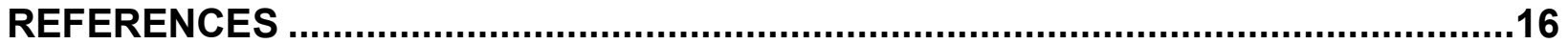




\section{Executive Summary}

An innovative combination of optical sensor technologies was explored with the objective of developing intelligent control systems for advanced coal combustion systems. The foundation of the project involves coupling mercury cavity ringdown spectroscopy (CRDS) with other monitoring techniques, such as, classical IR absorption spectroscopy (using tunable NIR/IR lasers) to provide real-time data on to monitor typical emission gases, (for example: $\mathrm{CO}_{2}, \mathrm{CO}$, $\mathrm{NO}, \mathrm{NO}_{2}, \mathrm{~N}_{2} \mathrm{O}, \mathrm{SO}_{2}$, and mercury). Such a combination of ultra-trace sensors and traditional sensors has the potential for providing the significantly increased sensitivity while maintaining the ability to work reliably in the hostile environment that will be required by next generation of coal-powered facilities.

Cavity ringdown spectroscopy has now reached the status of being of a mature analytical technique and, with respect to analytical atomic applications, a full discussion of recent can be found in refs 1-10. In earlier studies by the PI, ${ }^{4-11}$ a stand-alone CRDS system was used to determine the concentration of, for example, elemental mercury. That is, the system used a single cavity and provided a single output. In this study, a dual-cavity system was built and a Labview-based program developed to control and analysis the multiple cavities and analysis the output. Additional inputs can be accommodated with the addition of the appropriate VI's to expand analysis and control to a multichannel monitoring system. Although beyond the scope of the project, the long-term goal is to incorporate this system within data analysis and control functions of future generation coal-power utilities.

Two laser sources; a $10 \mathrm{~Hz}$, Nd-YAG-pumped dye laser emitting at 253nm and a NIR diode laser operating at $1520-1570 \mu \mathrm{m}$, were used as light sources. The output from the cavity was measured by the photodiode and the Photon Multiplier Tube (PMT) and transferred into the PC using fast analog-to-digital board (Gage 1250M). A Labview package was developed to perform data acquisition, analysis and control. This program acquires the multiple outputs from the cavity and displays the ring-down time and the concentration levels of the samples introduced into the cavity as function of the wavelength. The objective of this research project was the writing, implementation and testing of the Labview software to be used in Cavity Ring-down experiments for duel channel monitoring.

Our overall conclusion, from both this work and our previous studies, ${ }^{4-11}$ is that mercury CRDS remains the most promising technique to provide real-time monitoring of elemental mercury in exhaust gas streams. However, the UV linewidth of tunable light source must be sufficiently narrow to allow the differentiation of the mercury from the $\mathrm{SO}_{2}$ background and the repetition rate must be sufficiently high $(\sim 50 \mathrm{~Hz})$ to provide real-time measurements. Coupling this technique with the appropriate sampling system has the potential of playing a significant role in the development of intelligent control systems for advanced coal combustion systems. The design and construction of the dual-cavity system and the operational procedures of the control and analysis program are explained and the requirements for future implementation are discussed. 


\section{Experimental}

The multi-cavity system, laid out in this work centers around on an experimental technique that is known to able to provide the necessary sensitivity for mercury emissions required by next generation of coal-powered facilities. This technique is cavity ringdown spectroscopy (CRDS). CRDS is a sensitive absorption technique that was first developed by O'Keefe and Deacon in $1988^{1}$ and its application to atomic analytical problems dates from work began by the PI in $1997 .{ }^{9}$ This technique injects a pulse of light into a stable optical cavity formed by two highly reflecting mirrors. The light reflects back and forth in the cavity giving extremely long effective pathlengths. Using mirrors with a reflectivity of $99.99 \%$ and a 1 meter long cavity it is possible to achieve an effective pathlength of 10 kilometers. As the light reflects back and forth in the cavity a small amount of light is transmitted through the end mirror of the cavity to a photon detector such as a photomultiplier tube. The light exiting the cavity decays exponentially with time at a rate determined by round trip loss mechanisms within the cavity. The measured time constant for the exponential decay of light is called the "ringdown time" of the cavity. The dominant loss mechanism for an empty cavity is the reflectivity of the mirrors. However if a sample species, which absorbs light at a particular wavelength, is placed within the cavity the ringdown time will decrease from that of the empty cavity at that particular wavelength. The ringdown time is given by

$$
\tau=\frac{l_{c}}{c\left[(1-R)+\alpha l_{s}\right]}
$$

where, $l_{c}$ is the cavity length, $R$ is the reflectivity of the mirrors, $\alpha$ is the absorption coefficient of the sample species of interest, and $l_{s}$ is the pathlength through the sample. Once the empty cavity losses have been determined, CRD spectroscopy provides an absolute measure of the concentration of the absorbing sample within the cavity. This self-calibrating feature differentiates CRD from other highly sensitive laser-based methods such as laser-induced fluorescence (LIF) or resonantly enhanced multiphoton ionization (REMPI). In addition, this technique has been shown to have the ability to differentiate and measure the concentration of species that interfere with each other (e.g. $\mathrm{Hg}$ and $\mathrm{SO}_{2}$ ). For a full review of analytical atomic CRDS, see reference 5 .

\section{Experimental set up}

To establish a multichannel system, the following instrument (Figs. 1 and 2) was designed and built. The experimental setup consisted of two laser systems, a dual cavity setup, photo detector, photon multiplier tube, oscilloscope, PC, chopper, focusing lens, fiber optic cables and optical table. The laser sources that were available for the project were a $10 \mathrm{~Hz} \mathrm{Nd-YAG-pumped} \mathrm{laser}$ and a tunable NIR diode laser. The dye laser will produce pulse energies of $>0.5 \mathrm{~mJ} /$ pulse at 253 $\mathrm{nm}$, and have a linewidth of $0.03 \mathrm{~nm}$ at $503 \mathrm{~nm}$. This laser pulse was spatially filtered and mode matched to the cavity. The NIR diode laser is a commercially available tunable (1450-1650 nm) diode laser (New Focus). A chopper was used to tune the cavity (necessary due to the narrow linewidth) with diode laser. The experiments undertaken with both lasers used parallel cavities (see Figures 1 and 2) with identical cavity lengths $(60 \mathrm{~cm}$ ) and specially coated plano-concave

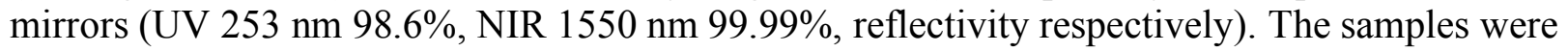
provided by calibrated sources. The cavity and supply lines were heated and insulated to prevent 

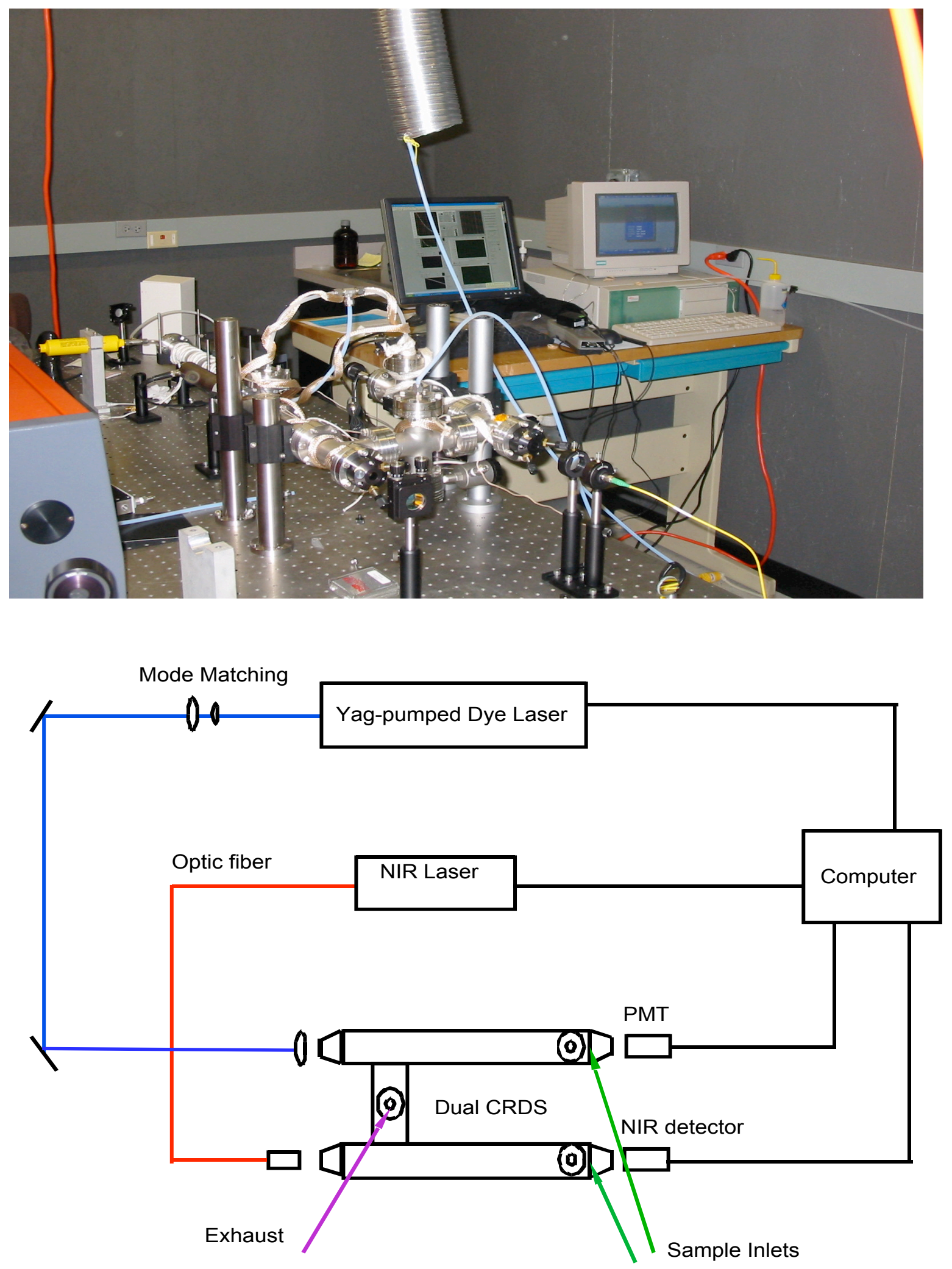

Figures 1 and 2: Top: Photograph of experimental layout (insulation removed). Bottom: A schematic layout of equipment in the photograph (rotated $90^{\circ}$ ). 
the samples from condensing on to the walls of the sample lines and chamber. To ensure uniform flow, the flow was split and introduced through two ports and exited through a vacuum pump via a single exit. The system pressure, controlled by flow controllers, was measured at the exit port.

The data acquisition system was designed around a LabView program run by a PC computer. The program controls the system and takes the output of the detectors, via a fast analog-to-digital 12-bit, two channel, $100 \mathrm{MHz}$ card (Gage) and analyzes the sample concentrations. This program was modified from earlier programs to handle the data acquisition and processing for a multichannel system. In addition, it also controls the detection systems (in this case, PMT, the lasers, etc). The program sets the experimental parameters, collects the data, calculates the decay time of the ringdown curve, self-calibrates, and determines the absolute concentrations of species of interest in the absorption cell at any time. In addition, the program is designed to be expandable to accept input from other types of sensors (e.g. $\mathrm{NO}_{\mathrm{x}}$ sensors, etc.) to allow the future development of a sensor network.

\section{Control and Analysis}

One of the main objectives of the project was to build a dual-cavity sampling system that could be used as a critical element in an optical offgas sensor network. Another major objective was to adapt Labview based control software to control the Cavity Ring-Down Spectroscopy (CRDS) system, as well as perform data acquisition and analysis. The first phase of this task was to identify the equipment to be controlled and the data required analyzed. The second phase was to analyze the input and output parameters, preset values and set the controls on the front panel of the main Virtual Instrument (V.I). The tasks also included development and analysis of sub V.I's to be used in the main V.I. The outputs of each sub V.I's would have to be tested individually and integrated under the main V.I. The final phase of this task was the layout of the front panel which contains the controls, indicators, input variables, graphs, and testing of the VI's using the signal generators. The project was then concluded with a test of the complete package using the experiment setup, verification and analysis of raw data. This task became the responsibility of the graduate student supported by this project and provided the basis of fulfilling his research requirements.

\section{Approach}

The control V.I's provided by the Gage software development kit (SDK) were first analyzed, tested and the necessary V.I's implemented. These V.I's are used to control the Gage oscilloscope and to retrieve data. Input and output parameters such as variables to be set by the end user, controls on the front panel, preset values and output format on the front panel are analyzed. The flow process of the data coming from the oscilloscope, the user input information and the calculations to be performed are recognized. Once the flow chart for the program development is known, each task is then implemented using Labview programming language. Programs or Sub VI's to perform each task such as determination of the fit of the curve, concentration calculations, etc are developed and tested. The main program consists of user controls, graphs and charts. The sub VI's, Gage oscilloscope VI's are integrated into the main VI. Initial troubleshooting was performed using a function generator analyzed and the functionality of the program. 


\section{Data Acquisition using Labview: Background}

Laboratory Virtual Instrument Engineering Workbench (LabVIEW) is a platform and development environment for a visual programming language. It is developed by National Instruments. LabVIEW is commonly used for data acquisition, industrial control and industrial automation on a variety of platforms using Microsoft Windows, UNIX, Linux and Mac OS. The programming language used in LabVIEW, called " $\mathrm{G}$ " is a dataflow programming language. Execution in LabVIEW is determined by the structure of a graphical block diagram in which different function nodes are connected by wires. These wires propagate data and any function node can execute as soon as all the input data is available. LabVIEW is capable of parallel execution using multi threading and multi processing hardware available on the host environment. In LabVIEW the user interfaces called the front panels are integrated into the development cycle. LabVIEW programs or subroutines are called Virtual Instrument (V.I's). Each V.I has three components: a block diagram, a front panel and a connector plane. Each individual V.I developed and tested can be further integrated or be used as a subV.I in a larger program. Controls and indicators on the front panel allow an operator to input data into or extract the output from a running Virtual Instrument. The main advantage of LabVIEW over other development environments is the extensive support for accessing instrumentation hardware. Drivers for different type of data acquisition hardware instruments are readily available for inclusion and can be integrated into the larger program. The LabVIEW development system allows creating stand-alone executables and the resultant executable can be distributed as it comes with all the necessary libraries. To summarize, Labview is used to perform control, data acquisition and analysis functions. It has been selected as the preferred programming language due to its unique front panel design, compatibility with Gage CompuScope LDK and its use in previous similar projects.

\section{Data Acquisition}

The signal from the photo detectors was converted into the digital format by a 12-bit A/D Gage card operating at $100 \mathrm{MHz}$, (the Labview programs to support this card are provided in the Gage software development kit). Once the signal is acquired and stored, the Labview program is triggered and the data is passed into the main program. Other parameters in the experiment are entered through the main tab of the Labview program.

The main parameters required to calculate the concentration of the sample inside the cavity are the length of the cavity, reflectivity of the mirrors, the values of the conversion factor and wavelength offset. The secondary parameters that influence concentration measurements are temperature and pressure. These are measured using a thermocouple inside the cavity. The lengths of both the cavities are entered into the main program. The percentage gate values to define the fit of the ringdown curve are also entered by the user.

The first phase of the project was finding the ringdown time for the cavity with no sample present. The flow of data from the cavity through various Virtual Instruments of Labview is shown below. The data from the oscilloscope is acquired from the Virtual Instruments (V.I's) from the Gage software development kit. 


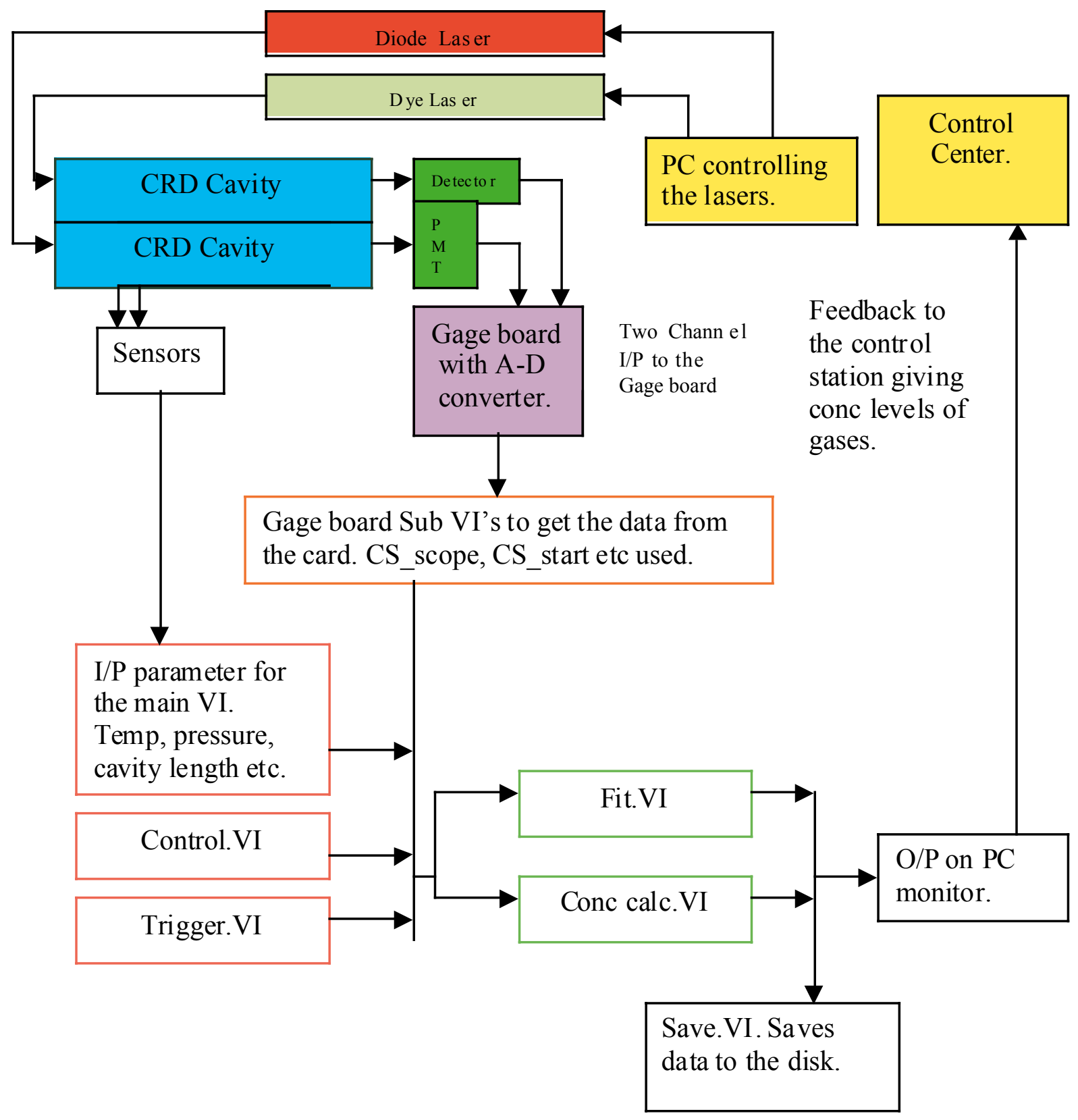

Figure 3: Control and Data Analysis Flow Schematic 


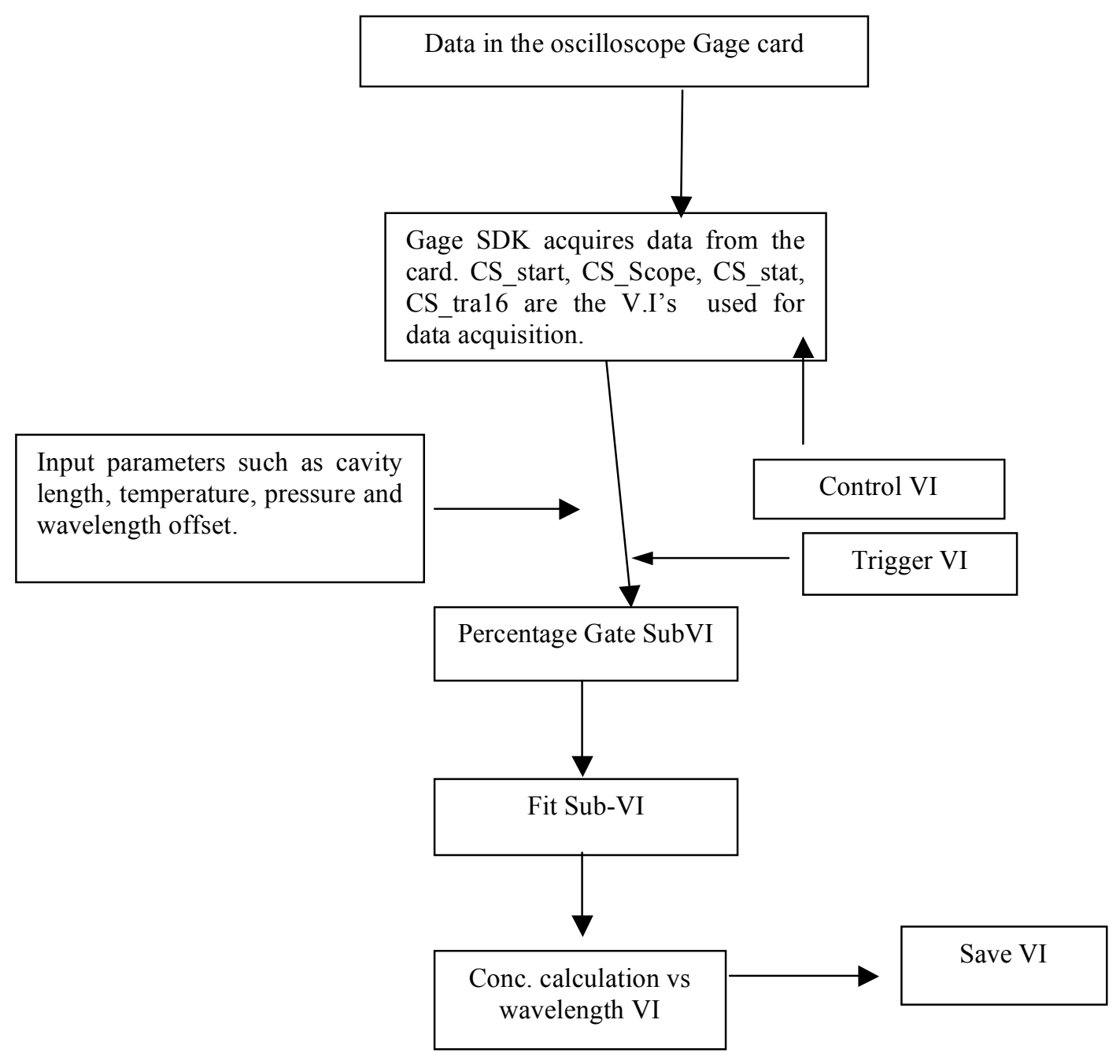

Figure 4: Schematic of the software control.

The mode of data capture, transfer rate, number of steps involved, nature of input and the range of the input data is controlled by the Control Sub VI. This V.I controls and triggers the data coming from the oscilloscope card. The input parameters such as cavity length, temperature, pressure, percentage gate values and the wavelength offset are entered through the main tab of the program. Once all the required data is acquired the calculation of the ringdown time and the concentration of the sample is performed. This is done by the sub V.I's percentage gate, fit and concentration calculation. These values of concentration are stored on the local PC using the Save V.I.

Once the parameters are all defined, the process follows the standard development path for all labview programs. For example, the concentration is calculated by the Concentration Calc VI. 


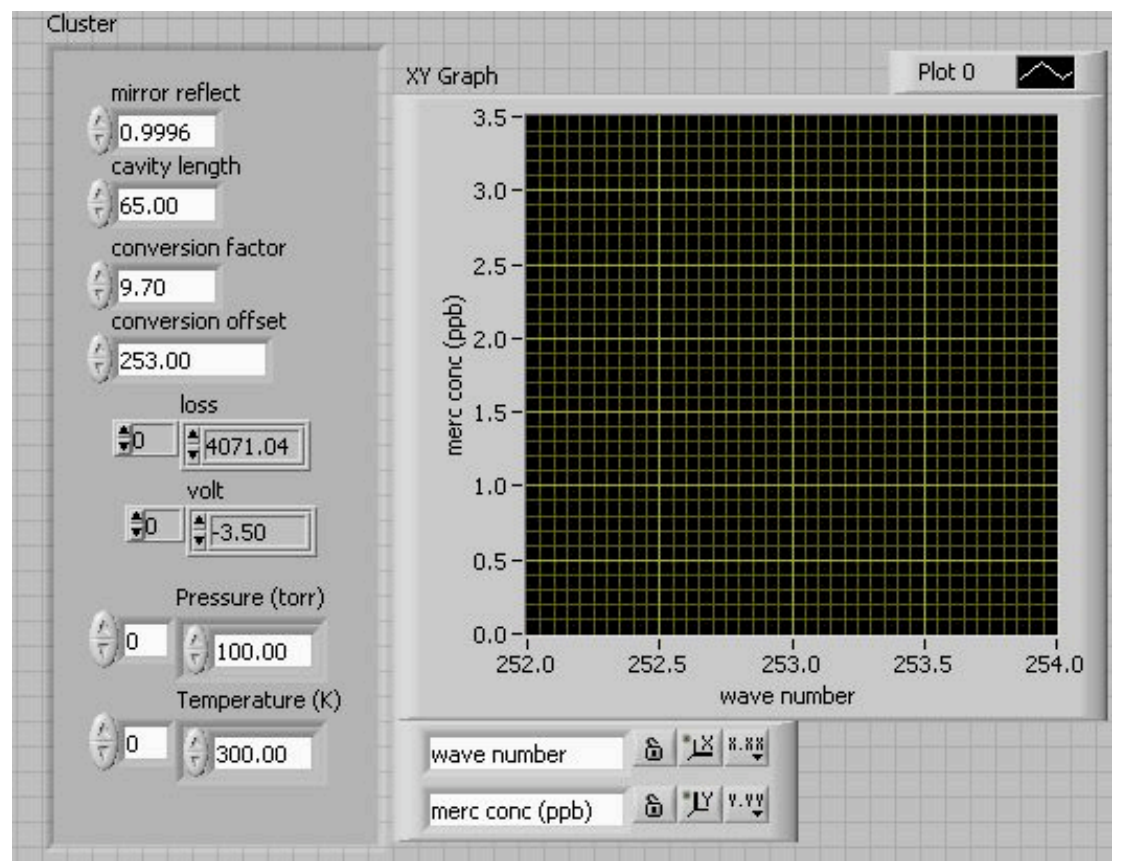

Figure 5: Concentration VI

This V.I is used to calculate the concentration of the sample and show the plot of concentration of the sample versus the wavelength or the wavenumber. The concentration is calculated using the parameters such as the length of the cavity, mirror reflectivity and the absorbance. The secondary parameters of temperature and pressure are also entered through this V.I. The output is shown on the main tab of the program.

The next plot shows the screen where an individual waveform is captured before they are averaged and sent to the plot described above. In this instance, the one channel is shown in white and a second channel is shown in green. Plots from multiple channels can be viewed simultaneously on this same graph and are used to monitor cavity performance. The example here shows an exponential decay curve for channel A.

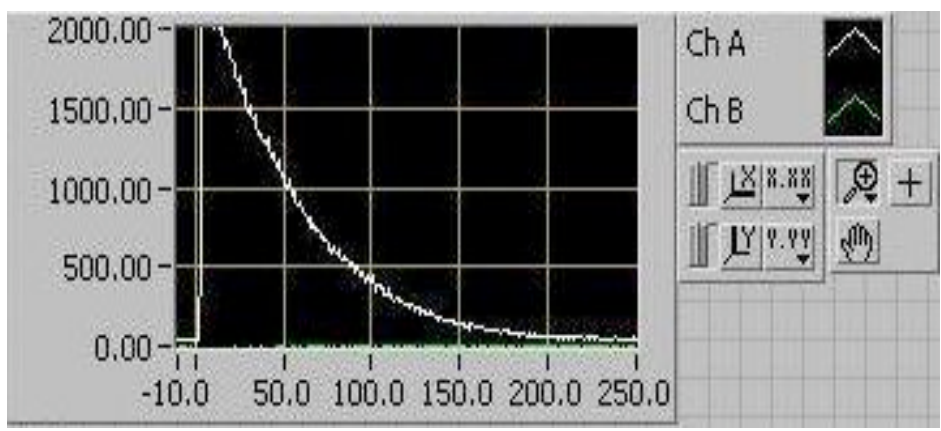

Figure 6: Individual waveform Plot 


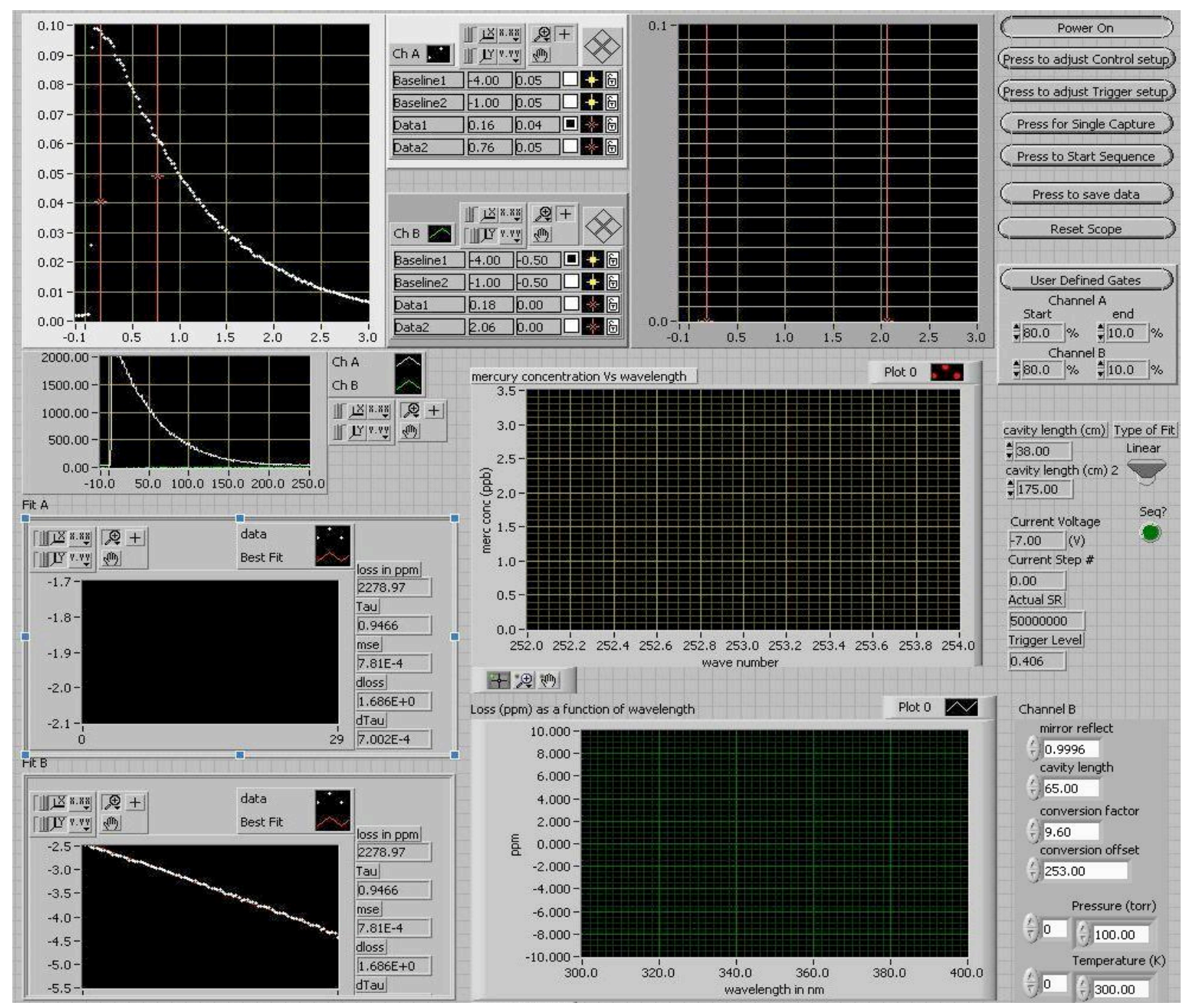

Figure 7: Main screen of the program.

The main screen, Figure 7, is where the user can view the data that is collected by the program, enter the values that are required, view the fit of the curves, save the data collected and control the experiment. The sub V.I's described in the previous section run in the background of this main program. Monitoring of multiple channels can be done through this main tab. Functions of each graph on the main tab are explained in the section below.

The graph below (Fig. 8) plots the averaged data taken from several traces of the data from the oscilloscope card. In order to apply the fit the area of the curve needs to be determined. From this program the user can input the percentage gate values for both the channels or can adjust the values by moving the cursors shown by the red lines on the graph. The number of points captured before the trigger is defined by the control setup that can be accessed by using the 'Press for control Setup' tab. The averaged data values both the channels can be viewed simultaneously using separate graphs on the main tab. 


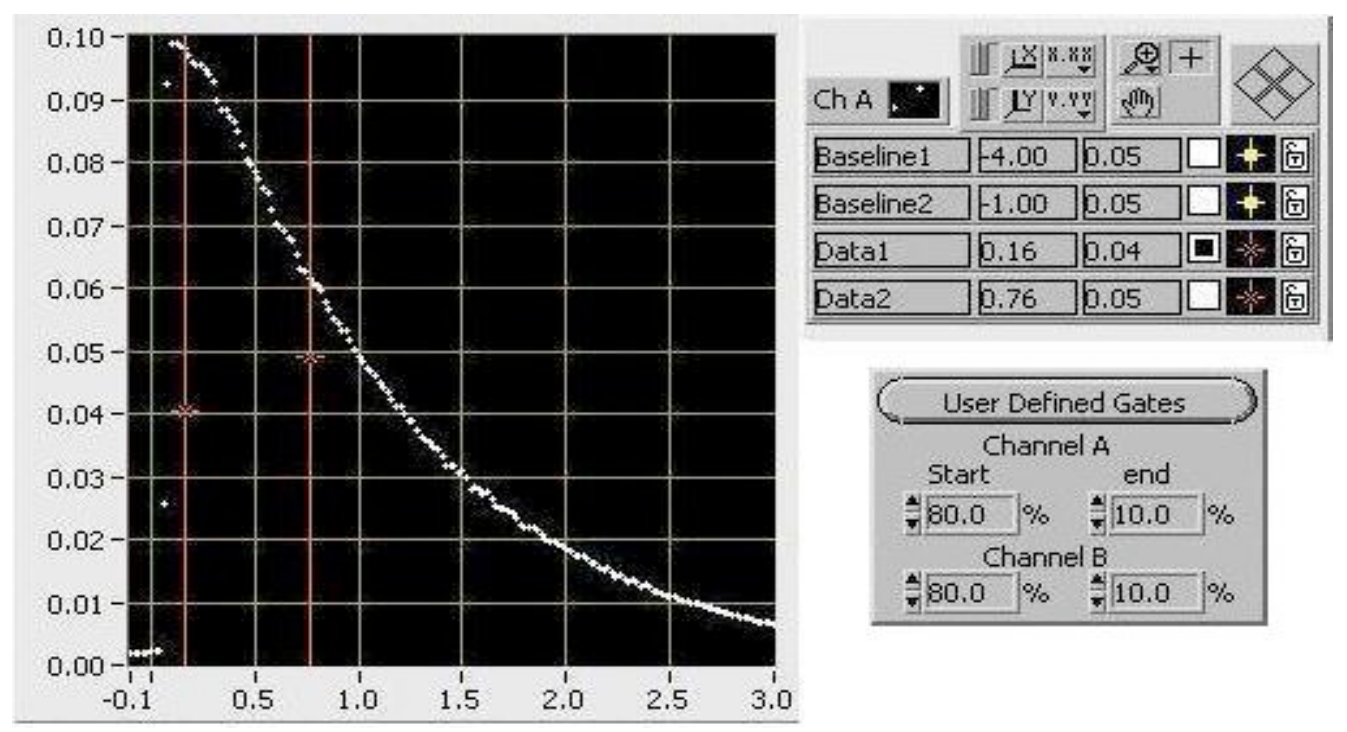

Figure 8: Averaged Signal

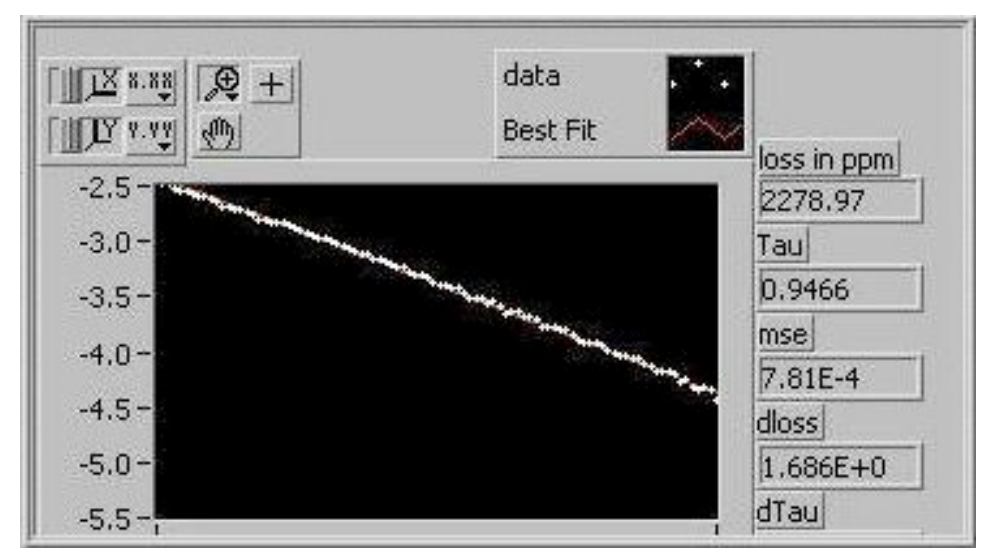

Figure 9: Exponential data fit

This plot in Figure 8 and 9 shows the curve and its corresponding exponential fit. The white dots indicate the data from the curve and the red line shows the best fit. This provides input on cavity alignment. The calculated loss parameters are shown on the side of the plot. The fit for both the channels can be viewed simultaneously by two separate graphs present on the main tab.

\section{Results and Discussion}

The dye laser is a reconditioned 1994 Scanmate 2E with integrated YAG pump laser and SHG Frequency Doubling Unit attached. The nominal linewidth, using the etalon accessory, was 0.03 $\mathrm{nm}$ at $503 \mathrm{~nm}$. To get the dye laser to produce the required $253 \mathrm{~nm}$ output, it was necessary to pump the dye using the $355 \mathrm{~nm}$ YAG and frequency double, This results in the broadening of the dye laser output linewidth to $\sim .1 \mathrm{~cm}^{-1}$. The other experimental concern was the low repetition rate, $10 \mathrm{~Hz}$, of dye laser 
Prior to upgrading the dye laser to operate in the $253 \mathrm{~nm}$ spectral range (mercury), we performed a high-resolution scan of $340 \mathrm{~nm}$ region (Figure 3). One scan consisted of averaging 100 ringdowns per step and took approximately four hours to complete and, as can be seen in Figure 10 , the $\mathrm{H}_{2} \mathrm{CO}$ band was not well-resolved. The cause was determined to be a combination of the required duration (low repartition rate), linewidth and overall beam quality. To put this performance in perspective, Figure 11 gives the results of a published (Wang, Mazzotti, Koirala, Winstead, and Miller. Appl. Spectrosc 58, 734, (2004)) CRDS scan, and its computer simulation, we obtained using a $20 \mathrm{~Hz} 0.04 \mathrm{~cm}^{-1}$ YAG-pumped dye laser. In those scans the high resolution scan clearly matched the computer simulated spectra. A performance level we have not been able to dulicate with the older laser.

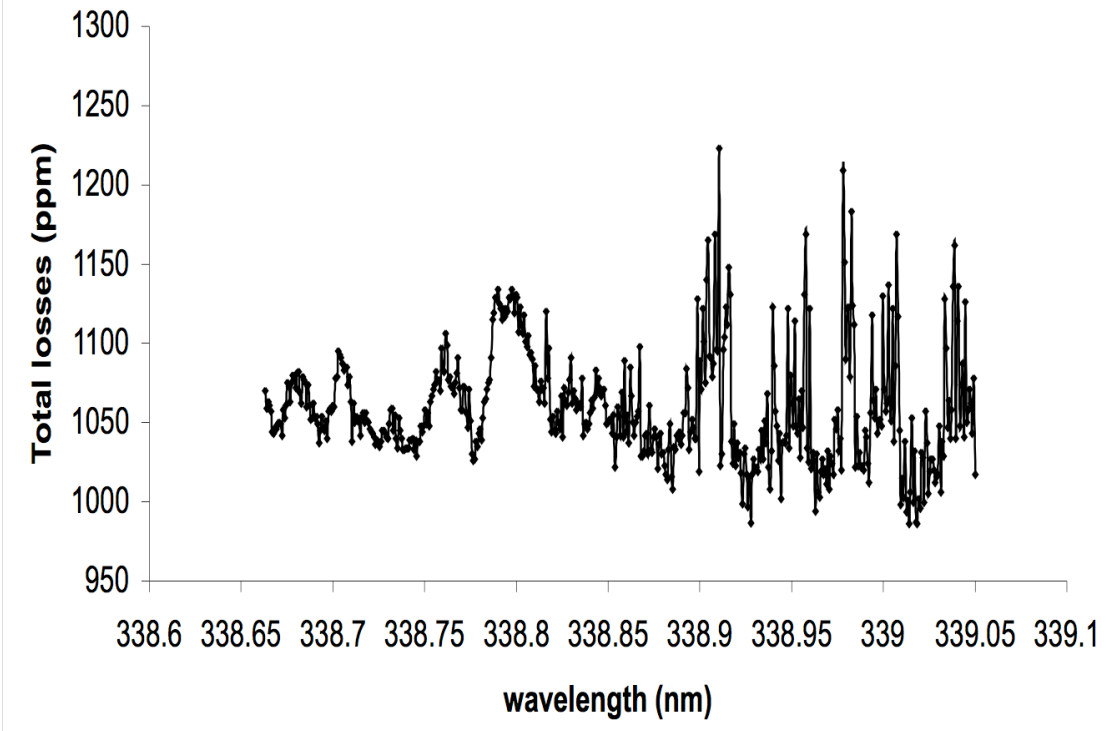

Figure 10: High resolution CRDS scan of $\mathrm{H}_{2} \mathrm{CO}$ band
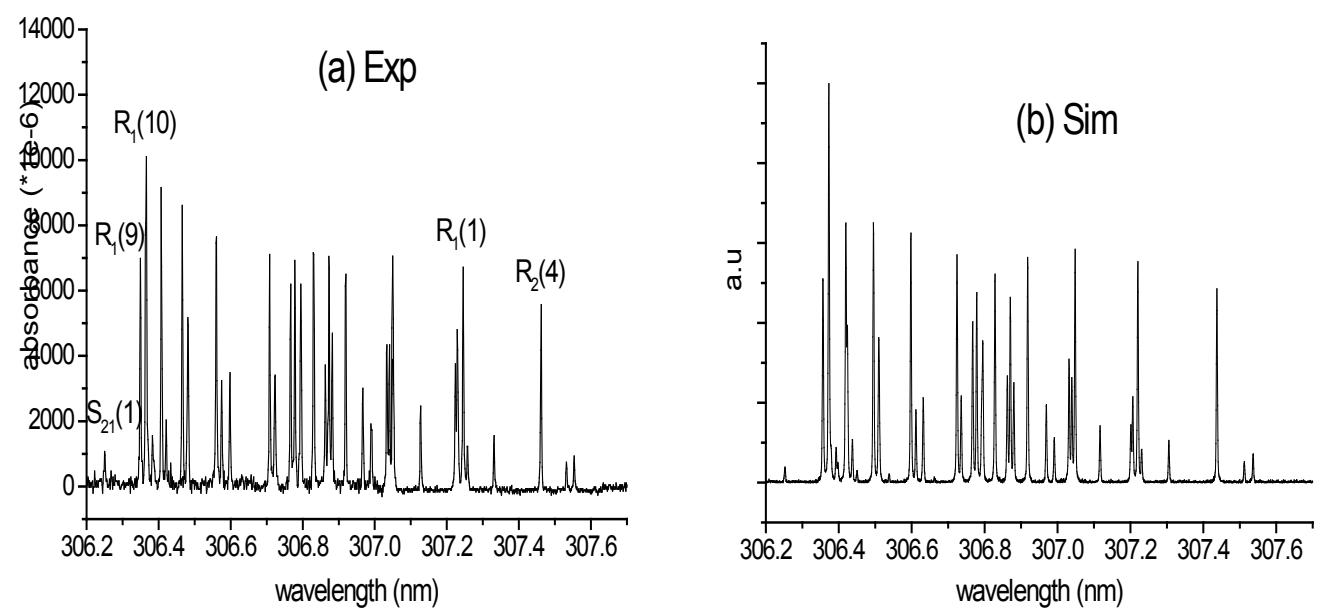

Figure 11: CRDS scan of OH radicals. See Figure 1, Applied Spectroscopy, 58734 (2004) 
As a result of this scan and further evaluation, it was determined that the dye laser did not have the performance characteristics necessary to permit mercury isotopic analysis. Therefore, no additional studies using $\mathrm{SO}_{2} / \mathrm{Hg}$ mixture were undertaken.

For multi-channel evaluation, measurement of another component of the offgas stream was required. Taking into account available light sources, it was determined that a carbon monoxide absorbance in the NIR was accessible. The use of CRDS for CO analysis could provide sub-ppm level monitoring. However, to achieve this a light capable of tuning across the fundamental $\mathrm{CO}$ absorption band $(\sim 4.5 \mu \mathrm{m})$ would be required. Although potential sources are available at this wavelength, for this project we were limited to the use of NIR tunable laser which meant only a weak CO absorption at $1560 \mathrm{~nm}$ was available. (FYI previous Quantum Cascade laser studies we have done at $4.5 \mu \mathrm{m}$ indicate that $\mathrm{LN}_{2}$ cooling is required to obtain the required beam quality). Taking into account cavity length etc, the available absorption feature, CO CRDS would provide a detection limit of the order of $20 \mathrm{ppm}$ at $1568 \mathrm{~nm}$. Figure 6 is a CRDS scan of CO at a concentration of approximately $60 \mathrm{ppm}$. No evident of interference from the present of elemental mercury (up to $240 \mathrm{ppb}$ ) in the gas stream was detectable.

\section{CO NIR CRDS}

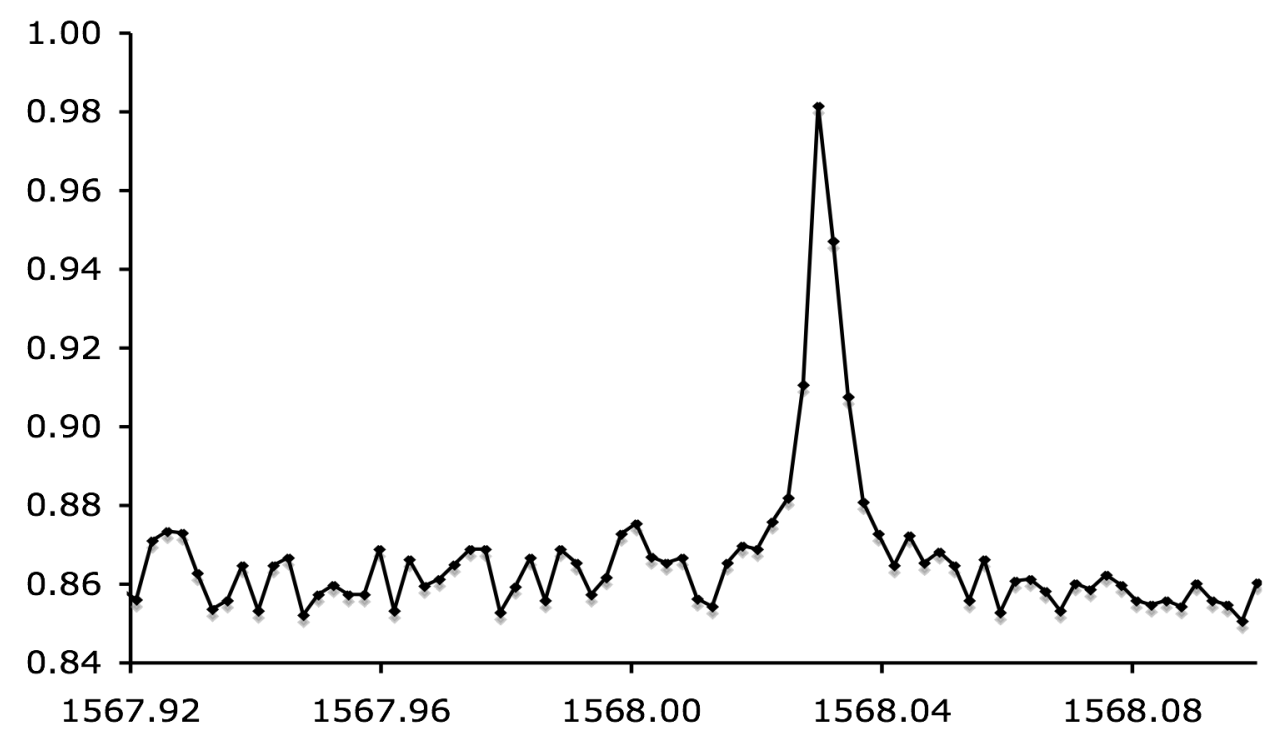

Figure 14: Absorbance (a.u.) versus wavelength (nm): A NIR CRDS scan of Carbon Monoxide

\section{Conclusions}

Cavity ringdown spectroscopy has reached the stage of a mature analytical technique. With the appropriate light source and highly reflective mirrors, it has the potential to provide real-time data that could be utilized in the control and monitoring functions of coal-powered utilities. For example, its ability to measure mercury concentrations at ppt levels in environments, including 
high levels of interferents such as $\mathrm{SO}_{2}$, make it the most promising technique presently available for mercury emission monitoring. ${ }^{10}$ It also has potential for measuring a number of other compounds of environmental interest and these studies are underway. However, I will confine my comments to Hg-CRDS. The underlying science is understood to the point that instrument development is effectively now an engineering problem. CRDS is laser-based and although laser technology has made significant progress in ease of use, beam quality and reliability in recent years, no breakthroughs have been made in the UV region of interest. To construct and deploy an Hg-CRDS instrument that will succeed in the real-world environment the following must be provided:

Light sources: As shown the old laser used above would not be the appropriate choice for a CRSD system. Hg-CRDS requires source having a narrow linewidth, $50 \mathrm{~Hz}$ (or higher), and that are tunable over several nanometers, centered at $253 \mathrm{~nm}$.

The choice of laser remains between

Dye lasers - stable, robust, produces waste (especially at high repetition rates).

Solid State - very narrow linewidth (if diode seeded), no wastes, more expensive (Diode lasers - Not available in UV)

Finally, all sources that meet CRDS requirements will likely require an environmentally controlled environment to some degree.

Sampling Interface: As far as I am aware, this has not specifically been addressed. However, an isokinetic sampling system based on one we developed and tested for an air-ICP multi-metal emission monitor would provide a good starting point. That system, which was invited to participate in a DOE/EPA demonstration, ${ }^{14}$ had a built-in method to establish and account for sample line losses.

Testing Facilities: Although a mature technique in the laboratory, the opportunity to rigorous field-testing the instrument will be critical to its successful deployment.

To conclude, despite its handicap of equipment cost and hands-on requirements, a CRDS-based mercury emission monitor remains, in my opinion, the most viable option for ultra-trace mercury monitor that will find a place in the sensor network for the next generation of coal-powered facilities.

\section{References}

1. O’Keefe and Deacon. Rev. Sci. Instrum. 59, 2544 (1988)).

2. Bush W. Kenneth and Marianna A. Busch. "Introduction to Cavity-Ringdown

Spectroscopy.” Cavity Ringdown Spectroscopy: An Ultratrace Absorption Measurement

Technique. Ed. K. W. Busch and M. A. Busch. ACS Symposium Series 720. American Chemical

Society, Washington D.C 1999. 
3. D. R. Wagner, C. B. Winstead, and G. P. Miller "Infrared Cavity Ringdown Spectroscopy", in Handbook of Vibrational Spectroscopy, J.M. Chalmers and P.R. Griffiths (Eds), John Wiley \& Sons, Ltd, Volume 1, pp. 866 - 880 (2002).

4. G. P. Miller and C. B. Winstead, Cavity Ringdown Laser Absorption Spectroscopy, pp10734-10750, Encyclopedia of Analytical Chemistry: Instrumentation and Applications. Edin-Chief Dr. Robert A. Meyers. John Wiley \& Sons Ltd. (2000). Updated chapter will appear in the 2008 issue.

5. Chuji Wang, Fabio J. Mazzotti, Christopher B. Winstead, and George P. Miller, Isotopic Measurement of Uranium Using ICP-Cavity Ringdown Spectroscopy Appl Spectrosc 57, 1167 (2003).

6. C. Wang, F. J. Mazzotti, C. B. Winstead, and G. P. Miller. "ICP-CRDS - Cavity Ringdown Spectroscopy as a plasma diagnostic.” Applied Spectroscopy. 56, 386 (2002).

7. S. Tao, F. J. Mazzotti, C. B. Winstead, and G. P. Miller, "Determination of elemental mercury by cavity ringdown spectrometry" Analyst 125, 1021 (2000).

8. Fabio J. Mazzotti Investigation of inductively coupled plasma as an atomization source for analytical and fundamental measurements using cavity ringdown spectroscopy. $\mathrm{PhD}$ Thesis (2004).

9. G. P. Miller and C. B. Winstead. ICP-Cavity Ringdown Spectroscopy. J. Anal. Atomic Spectra 9, 907 (1997).

10. Final Technical Report "A cavity ringdown spectroscopy mercury continuous emission monitor” DE-FC26-01FT41221. Sensor research \& Development Corp. (2004).

11. United States Environmental Protection Agency. “Controlling Power Plant Emission Overview” Nov, 2007. http://www.epa.gov/mercury/control_emissions/index.htm. 
12. United States Environmental Protection Agency. "Regulatory Actions" Oct 11, 2007. $<$ http://www.epa.gov/air/mercuryrule/rule.htm> .

13. Labview: The software that powers Virtual Instruments. www.ni.com,

14. G. P. Miller, Test Results from the DOE/EPA sponsored Multi-metals Continuous Emission Monitor Demonstration. A Portable ICP-AES System for on-Line Monitoring of Toxic Metals. Appendix B1-30 pages. 1997 Performance Testing of Multi-Metals Continuous Emissions Monitors DOE/ID-10665 September 1998. 